\title{
Cavernosopatía traumática crónica. Un nuevo síndrome de fibrosis peneana
}

\section{Chronic Traumatic Cavernopathy. A New Syndrome of Penile Fibrosis}

\author{
Juan F. Uribe A. ${ }^{1}$ Alejandro Vélez H. ${ }^{2}$ John J. Zuleta T. ${ }^{3}$ Carlos A. Uribe T. ${ }^{4}$ \\ ${ }^{1}$ Servicio de Urología, Hospital Pablo Tobón Uribe, \\ Medellín, Colombia \\ 2 Servicio de Patología, Hospital Pablo Tobón Uribe, \\ Medellín, Colombia \\ ${ }^{3}$ Servicio de Epidemiología, Hospital Pablo Tobón Uribe, \\ Medellín, Colombia \\ ${ }^{4}$ Servicio de Medicina Sexual, Hospital Pablo Tobón Uribe, \\ Medellín, Colombia \\ Urol Colomb 2018;27:156-166. \\ Address for correspondence Juan F. Uribe A., MD, Servicio de \\ Urología, Hospital Pablo Tobón Uribe, Medellín, Colombia \\ (e-mail: medicinasexual@une.net.co; urologiahptu@gmail.com).
}

\section{Resumen \\ Palabras Clave \\ - enfermedad peneana \\ - peyronie \\ - induración peneana \\ - traumas de pene \\ - disfunción eréctil \\ - fibrosis \\ - ultrasonografía}

Introdución La llamada genéricamente "enfermedad de Peyronie» es un diagnóstico que explica un porcentaje de ciertos estados de fibrosis peneana grave, pero se queda corta para abarcar la amplia gama de grises de la fibrosis peneana secundaria a microtrauma sexual repetido.

Objectivo Describir un nuevo síndrome por trauma repetido en el pene al que se propone denominar "cavernosopatía traumática crónica» (CTC), con 4 componentes: fibrosis (leve, moderada o grave), disfunción eréctil, curvatura y fugas venosas peneanas.

Materiales y métodos Se realizó un estudio de corte transversal en una base de datos de 128 pacientes que tenían al menos una ecografía de pene con vasoactivo y en los que se había detectado algún grado de fibrosis cavernosa según la clasificación de Levine. Se evaluó la presencia de disfunción eréctil, curvas y fugas venosas según el grado de fibrosis. El estudio contó con la aprobación del Comité de Ética en Investigación.

Resultados Se estudió a 128 pacientes con fibrosis, con 51,3 años de edad promedio $(D E=13)$. El grado de fibrosis fue leve (Levine 1 ) en $30(23,43 \%)$, moderado (Levine 2 ) en $23(17,96 \%)$ y grave (Levine 3 ) en $75(58,6 \%)$. Se demostró que, a mayor fibrosis, mayor disfunción eréctil, 66,6; 83,6 y 96\% para Levine 1, 2 y 3, respectivamente $(p \leq 0,0001)$. La frecuencia de curvatura secundaria $(n=71)$ fue de $0 ; 4,2$ y $95,7 \%$ para Levine 1,2 y 3 , respectivamente $(p \leq 0,0001)$. La frecuencia en los 44 pacientes con fugas cavernosas fue de $9 ; 20,4$ y $70,4 \%(p=0,0060)$ y en los 15 de fugas dorsales fue de $80 ; 13,3$ y $6,6 \%(p<0,0001)$ para Levine 1,2 y 3 respectivamente. El grupo de 79 pacientes $(64,03 \%)$ con mayor posibilidad de CTC, con presencia de 3 o 4 criterios que incluyeran curvatura secundaria y fuga cavernosa, tuvo un promedio de edad de received

September 4, 2016

accepted

February 2, 2017

published online

January 10,2018
DOI https://doi.org/

10.1016/j.uroco.2017.02.001.

ISSN 0120-789X.

eISSN 2027-0119.
Copyright $($ 2018, Sociedad Colombiana License terms

de Urología. Publicado por Thieme Revinter Publicações Ltda., Rio de Janeiro, Brazil. Todos los derechos reservados.

()(1) $\Theta \circledast$ 


\section{Abstract}

\section{Keywords}

- penile disease

- peyronie's disease

- penile induration

- penile injuries

- erectile dysfunction

- fibrosis

- ultrasound
$59,2$ años ( $D E=8,7)$, comparado con 41,7 años $(D E=11,7)$ en el grupo de menor posibilidad, diferencia estadísticamente significativa $(p \leq 0,0001)$.

Conclusiones Proponemos que existe un síndrome de microtrauma repetido en el pene, que puede denominarse CTC, análogo a la encefalopatía traumática crónica, con 4 componentes: fibrosis cavernosa, disfunción eréctil, curvatura peneana y fugas venosas. La fibrosis es un continuum que el paciente recorre por fases leves y moderadas, a menudo inapreciables para los clínicos. Las curvaturas secundarias y las fugas cavernosas están relacionadas directamente con el grado de fibrosis y con mayor riesgo de disfunción eréctil. La edad empeora algunos factores de la CTC.

Introduction The condition called "Peyronie's disease" is a diagnosis that explains a percentage of the states of severe penile fibrosis, but not enough to cover the wide grey range of penile fibrosis secondary to repeated sexual micro-trauma.

Purpose To describe a new syndrome due to repeated trauma to the penis, which is proposed to be called "Chronic traumatic cavernopathy" (CTC), with 4 components: fibrosis, erectile dysfunction, curvature and penile venous leakage.

Materials and Methods A cross-sectional study was conducted on a database of 128 patients who had at least one penis ultrasound performed with a vasoactive, and had some degree of cavernous fibrosis according to Levine classification. An evaluation was made on the presence of erectile dysfunction, curvature, and venous leakage depending on the degree of fibrosis. The study was approved by the Research Ethics Committee.

Results The study included 128 patients with penile fibrosis, and a mean age of 52.9 years $(S D=13)$. The degree of fibrosis was mild (Levine 1$)$ in $30(23.43 \%)$, moderate (Levine 2) in 23 (17.96\%), and severe (Levine 3 ) in 75 (58.6\%). It was shown that the higher the fibrosis, the more erectile dysfunction (ED), with $66.6 \%, 83.6 \%$, and $96 \%$ for Levine 1,2 , and 3 respectively $(P \leq 0.0001)$. Frequency of secondary curvature $(n=71)$ was $0 \%, 4.2 \%$, and $95.7 \%$ for Levine 1,2 , and 3 , respectively $(P \leq 0.0001)$. The frequency in 44 cavernous patients with venous leakages was $9 \%, 20.4 \%$, and $70.4 \%(P=0.0060)$, and with 15 dorsal leakages it was $80 \%, 13.3 \%$, and $6.6 \%(P<0.0001)$ for Levine 1,2 , and 3, respectively. The group of 79 (64.03\%) patients with increased possibility of CTC, and who met the 4 criteria, or the 3 criteria including secondary curvature and cavernous venous leakage, had a mean age of 59.2 years $(S D=8.7)$, compared to 41.7 years $(S D=11.7)$ in the other group with less possibility, which had a statistically significant difference $(P \leq 0.0001)$.

Conclusions We propose that there is a syndrome of repeated micro-trauma in penis, which can be called CTC, analogous to chronic traumatic encephalopathy, with 4 components: cavernous fibrosis, erectile dysfunction, penile curvature, and venous leak. Fibrosis is a continuum that, in the patient goes through mild and moderate stages, and often goes unnoticed by clinicians. Secondary curvatures and cavernous leaks are directly related to the degree of fibrosis and increased risk of erectile dysfunction. Age aggravates some factors of CTC.

\section{Introducción}

La llamada genéricamente «enfermedad de Peyronie» es un diagnóstico que incluye los estados de fibrosis peneana grave que comprometen la túnica albugínea, con o sin compromiso del tejido intracavernoso, pero cuyo concepto se queda corto para abarcar la amplia gama de otros tipos de fibrosis peneana que no afectan a dicha túnica, relacionados o no con trauma durante la actividad sexual. Dichos procesos de fibrosis podrían comenzar incluso desde el inicio de la vida sexual, sin alcanzar a reunir los criterios clínicos para denominarse enfermedad de Peyronie, tal como fue descrita por el ilustre barbero francés, y mucho menos los ultrasonográficos o histológicos de que disponemos actualmente. ${ }^{1}$

La llamada enfermedad de Peyronie, diagnóstico subyacente en los casos más graves de fibrosis peneana que afectan la 
túnica albugínea, fue descrita por el cirujano-barbero francés Francoise Gigot de La Peyronie en un tratado sobre trastornos de la eyaculación que data de 1743. Su epónimo fue el que trascendió en la historia, aunque existen múltiples referencias previas de la literatura que no lograron dar nombre a la enfermedad: correspondencia de Vesalio a Gabriele Falopio en 1561; una crónica del anatomista italiano Giulio Cesare Aranzi y otra del médico italiano Teodorico de Borgognoni, hablando en uno de sus tratados de «tubérculos en el pene» en 1267. 2

No existe una denominación unificada para las diferentes fases de la enfermedad fibrosa, tempranas o avanzadas con compromiso extratunical, y es inexplicable por qué, si está definido el microtrauma sexual repetido (MSR) como desencadenante de la fibrosis en una enfermedad con tan amplia gama de grises, la literatura sigue llamando Peyronie a condiciones que no lo son por definición y por qué se carece de un nombre apropiado para las otras entidades fibrosas, aun aceptando que ambos grupos son expresiones de 2 enfermedades distintas, aunque posiblemente relacionadas por factores inmunes, bioquímicos o genéticos. ${ }^{3-11}$

Haciendo una analogía con otras especialidades, podría hablarse de una "cavernosopatía traumática crónica» (CTC), tal como se ha demostrado con el hallazgo de una enfermedad semejante denominada chronic traumatic encephalopathy (encefalopatía traumática crónica [CTE]), descrita por el patólogo forense nigeriano Bennet Omalu, célebre actualmente por la película "Concussion". La CTE tiene un cuadro neuropatológico típico con placas amiloides difusas, ovillos neurofibrilares dispersos e hilos neuríticos taupositivos en áreas neocorticales, que causan clínicamente graves problemas cognitivos y que se encontró inicialmente en jugadores de fútbol americano, pero que se ha ido extendiendo, como teoría, a otros atletas y luego a otras profesiones que implican el microtrauma repetido. ${ }^{12,13}$

La propuesta es incluir una CTC como un nuevo síndrome, cuyos precisos componentes anatomopatológicos e inmunohistoquímicos están por develar, y con 4 componentes clínicos, a saber: fibrosis cavernosa, fallas en la erección, curvaturas secundarias y fuga venosa, en la que la llamada enfermedad de Peyronie sería un tipo de fibrosis peneana grave, que afecta obligatoriamente la túnica albugínea, pero posiblemente diferente de la CTC. ${ }^{14}$

\section{Objectivo}

Describir un nuevo síndrome por microtrauma repetido (MSR) en el pene al que se propone denominar CTC, con 4 componentes clínicos: fibrosis (leve, moderada o grave), disfunción eréctil, curvatura y fugas venosas peneanas.

\section{Materiales y Métodos}

Se realizó un estudio de corte transversal a partir de los registros de la atención clínica almacenados en una base de datos de una consulta de medicina sexual. El protocolo del estudio fue aprobado por el Comité de Ética en Investigación del hospital Pablo Tobón Uribe y se implementó respetando los principios éticos en investigación en humanos. Se seleccionó a 128 pacientes que cumplían con los criterios de inclusión. Todos los pacientes habían sido tratados mediante ecografía dopler high definition de pene con vasoactivo (prostaglandina E1 o mezcla de prostaglandina, papaverina y fentolamina-trimix), por el mismo examinador usando el mismo equipo (Voluson E8 General Electric). ${ }^{15}$ En el grupo de pacientes se había realizado una clasificación ecográfica de su grado de fibrosis usando las clasificación de Levine (grado 1-leve [LI], grado 2-moderada [L2], grado 3-grave [L3]), que es uno de los criterios de inclusión (-Figs. 1-3). Todos los pacientes tenían una pesquisa sobre disfunción eréctil, considerándose disfuncional con cualquiera de estas 2 escalas: un puntaje menor a 21 puntos en la escala del Sexual Health inventory for men (SHIM) o haber respondido negativamente a la pregunta sobre calidad de la erección en las últimas 4 semanas según la Erection Hardness Score (EHS). También en el grupo de pacientes se había verificado la presencia o no de curvaturas peneanas mayores de 30 grados, que se dividieron en primarias (desde el comienzo de la vida sexual) y secundarias (que aparecieron luego en la vida sexual). En el análisis estadístico se incluyó el universo de historias clínicas de los pacientes que cumplieron los criterios de
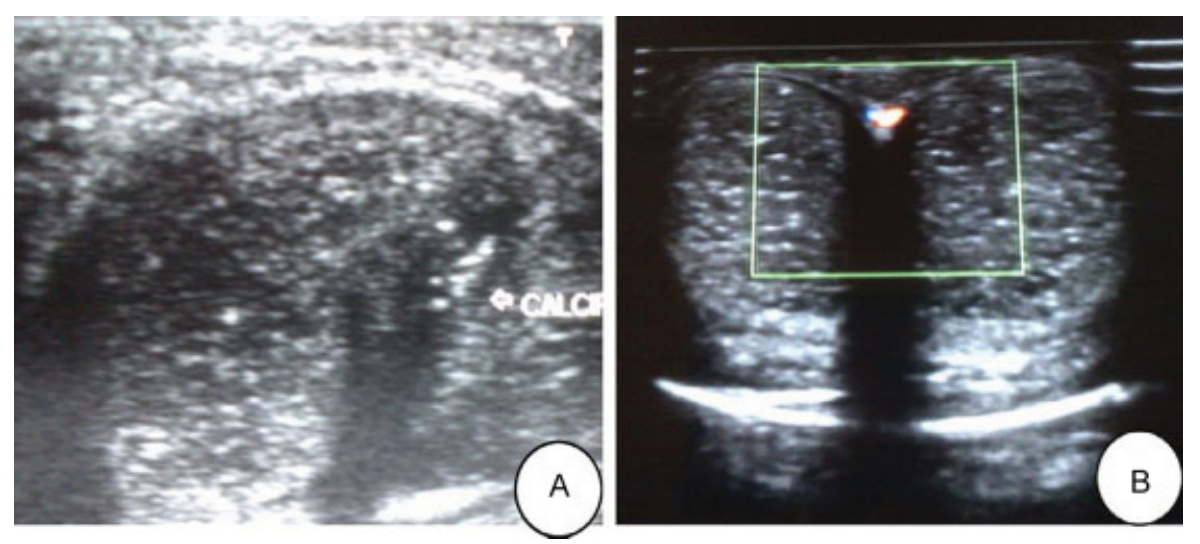

Fig. 1 Fibrosis leve (grado 1 de Levine). A) Punteado difuso y confluente. Calcificaciones menores de 0,3 mm, algunas con sombra ecogénica. No hay placas ni nódulos. B) Punteado difuso con calcificaciones menores de $0,3 \mathrm{~mm}$, algunas con sombra ecogénica. Se observa fuga venosa dorsal profunda. No hay placas ni nódulos. 11,18 

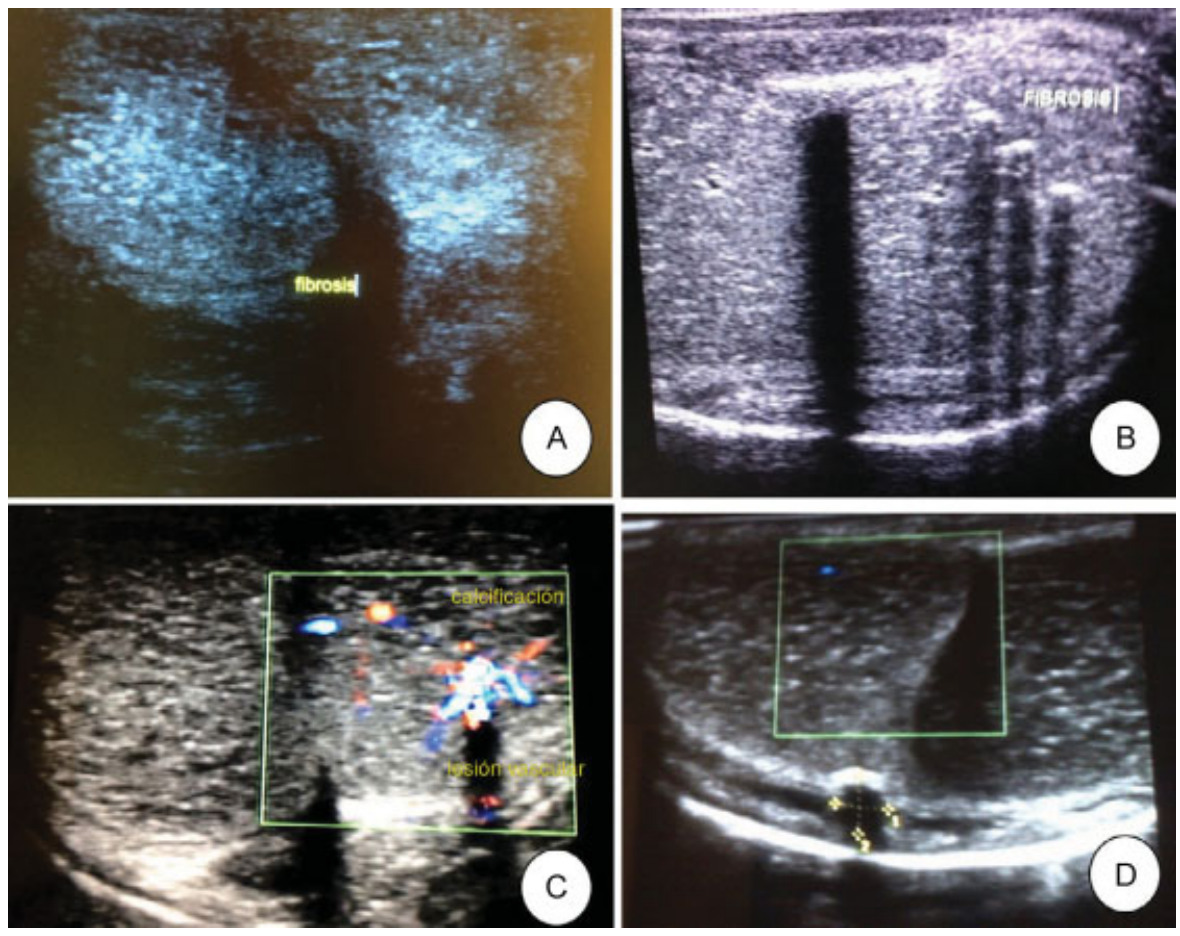

Fig. 2 Fibrosis moderada (grado 2 de Levine). A) Punteado confluente denso; calcificaciones menores de 1,5 cm. No hay placas ni nódulos. B) Calcificaciones múltiples pero menores de $1 \mathrm{~cm}$ con sombra ecogénica. C) Fuga cavernosa vecina a una zona de fibrosis. D) Placa periférica única menor de $1,5 \mathrm{~cm} .^{11,18}$
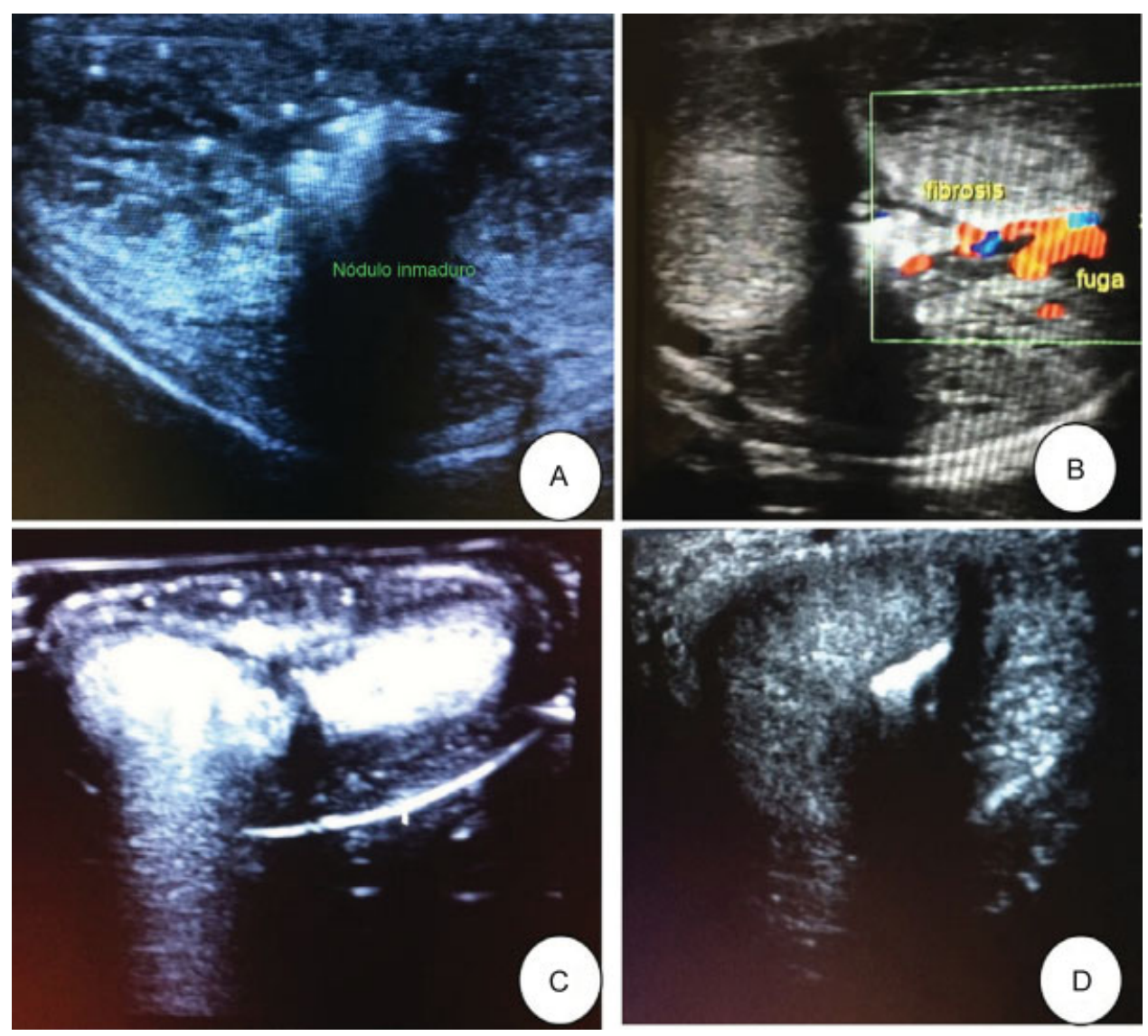

Fig. 3 Fibrosis grave (grado 3 de Levine). A) Placa inmadura, con bordes irregulares. B) Fuga cavernosa índice, vecina a una zona de fibrosis grave. C) Fibrosis cavernosa grave que ocupa ambos cuerpos cavernosos. D) Placa central calcificada en túnica albugínea mayor de 15 mm, típica de enfermedad de Peyronie. ${ }^{11,18}$ 
inclusión y exclusión. El resumen de las variables cualitativas se presenta con número absoluto y porcentaje; la edad se presenta con media y desviación estándar. La fibrosis como factor de exposición se dividió en 3 categorías (leve, moderada, grave), se evaluó la asociación con los otros factores mediante la prueba chi cuadrado de tendencia y se calculó la fuerza de asociación mediante la razón de prevalencias con su respectivo intervalo de confianza del $95 \%$, teniendo como grupo de referencia los pacientes con fibrosis leve.

\section{Criterios de Inclusión}

- Tener una ecografía dopler peneana como parte de un estudio de impotencia o de enfermedad de Peyronie. ${ }^{16,17}$

- Hallazgos de algún grado de fibrosis durante la ecografía dopler (con o sin: compromiso de la túnica albugínea, curvatura peneana, disfunción eréctil o fugas venosas). En este estudio no se excluyó a los pacientes con enfermedad de Peyronie. ${ }^{18}$

\section{Criterios de Exclusión}

- Origen de la fibrosis traumático externo.

- Antecedentes de fractura de pene y todos los traumas mayores de pene.

- Antecedentes de trauma de pelvis o uretra.

- Antecedentes de reconstrucciones peneanas por causas congénitas o estéticas.

- Uso continuado por más de 6 meses de medicamentos vasoactivos cavernosos.

\section{Clasificación ecográfica de la fibrosis}

Una clasificación ecográfica del grado de calcificación fue publicada por el Dr. Laurence Levine (2013) y revisada recientemente en Campbells Urology 11. ${ }^{\mathrm{a}}$ ed. (2016). ${ }^{11}$ Aunque otras modalidades de imagen se usan también para identificar placas calcificadas u osificadas, nosotros estamos de acuerdo con Levine en que la ecografía es el mejor método, relativamente barato, no invasivo y confiable, para la detección, no solo de placas, sino de cualquier tipo de fibrosis peneana. Las áreas de calcificación fueron definidas como regiones hiperecoicas con la presencia de sombra acústica usando los 3 grados ecográficos propuestos por Levine et al.: grado 3 , (placas $>1,5 \mathrm{~cm}$ en cualquier dimensión o múltiples placas $\geq 1,0 \mathrm{~cm}$ ); grado 2 (lesiones de $0,3 \mathrm{~mm}$ a $1,5 \mathrm{~cm}$ ) y grado 1 (lesiones $<0,3 \mathrm{~mm}$ ). ${ }^{18-22}$

\section{Resultados}

El presente estudio encontró un grupo de 128 pacientes con fibrosis, con 52,9 años de edad en promedio ( $D E=13,03$ ); de ellos, 30 pacientes $(23,43 \%)$ eran leves o Levine grado 1,23 (17,96\%) eran moderados o Levine grado 2 y $75(58,59 \%)$ eran graves o Levine grado 3 (-Fig. 4). Un 86,71\% (111) de los pacientes tenían algún grado de disfunción eréctil.

El estudio demostró en diferentes comprobaciones que, a mayor fibrosis (grave o L3), mayor disfunción eréctil, tanto

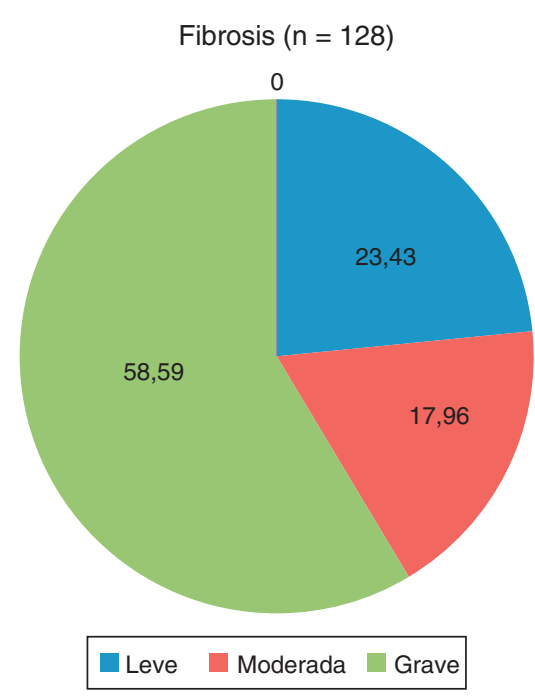

Fig. 4 Grados de fibrosis en el grupo completo.

cuando se escogieron solo los disfuncionales $(n=111)$, grupo en que la fibrosis leve (L1) fue de $18,01 \%$, la moderada (L2) fue de $17,11 \%$ y la grave (L3), de $64,86 \%$, como cuando se consideró cada nivel de fibrosis como un universo aparte $(\mathrm{n}=128)$ : leve o L1 $(66,6 \%)$, moderada o L2 (83,6\%) y grave o L3 (96\%). El riesgo de tener disfunción eréctil en fibrosis grave (L3) fue un $44 \%$ mayor que en fibrosis leve (L1) (-Tabla 1) (-Fig. 5).

Con respecto a las curvaturas, se encontraron 86 pacientes (67,18\%), de los cuales 15 (11,71\%) tenían curvaturas primarias y 71 (55,46\%) tenían curvaturas secundarias. La frecuencia de curvatura secundaria ( $\mathrm{n}=71$ ) fue de $0 ; 4,2$ y 95,7\% para los grado de fibrosis de L1, L2 y L3 respectivamente (p de tendencia: $<0,0001$ ) (-Tabla 2) (-Figs. 6 y 7).

Con respecto a las fugas venosas, el 53,2\% de los pacientes no tenían fugas. Del $46,08 \%$ restante, se encontraron 44 pacientes $(34,37 \%)$ con fugas cavernosas y 15 pacientes $(11,71 \%)$ con fugas dorsales ( - Tabla 3). De los 15 pacientes con fugas dorsales, 12 (80\%) cursaban con L1 (leves), 2 pacientes $(13,3 \%)$ con L2 (moderada) y un paciente $(6,6 \%)$ con L3 (grave); mientras que de los 44 pacientes con fugas cavernosas, 4 (9\%) cursaban con L1, 9 (20,4\%) con L2 y 31 (70,4\%) con L3 (-Figs. 8 y 9 ).

La - Tabla 4 resume los hallazgos encontrados con respecto a disfunción eréctil, curvaturas y fugas venosas según los diferentes grados de fibrosis.

Un concepto interesante es el de mayor y menor posibilidad de CTC. Se consideró de mayor posibilidad para el diagnóstico de CTC a aquellos pacientes que cumplían, o bien con los 4 criterios o al menos con 3, si estos incluían tener fugas cavernosas o curvaturas secundarias. El resultado fue que con 4 criterios se encontraron 27 pacientes $(21,07 \%)$ y con 3 criterios, 55 pacientes (42,96\%). Todos los otros se consideraron de baja posibilidad de CTC (46 pacientes $=35,97 \%)($ - Tabla 5) (-Fig. 10).

El grupo de "alta posibilidad de CTC" con 82 pacientes $(64,03 \%)$ tuvo un promedio de edad significativamente 
Tabla 1 Cavernosopatía traumática crónica con sus 4 componentes

\begin{tabular}{|c|c|c|c|c|c|c|c|}
\hline Fibrosis & $\begin{array}{l}\text { Edad en años } \\
\text { (fibrosis) }\end{array}$ & $\begin{array}{l}\text { Disfunción } \\
\text { eréctil }\end{array}$ & $\begin{array}{l}\text { Edad en años } \\
\text { (disfunción } \\
\text { eréctil) }\end{array}$ & Curvas & $\begin{array}{l}\text { Edad en años } \\
\text { (curvaturas) }\end{array}$ & Fugas & $\begin{array}{l}\text { Edad en años } \\
\text { (fugas) }\end{array}$ \\
\hline \multirow[t]{9}{*}{$\begin{array}{l}\text { Grave Levine } 3 \\
75(58,5 \%)\end{array}$} & 57,8 & $72 / 75$ (96\%) & 58,4 & $\begin{array}{l}\text { Curvatura } \\
\text { primaria 5/75 } \\
(6,66 \%)\end{array}$ & 41,4 & $\begin{array}{l}\text { Fuga } \\
\text { cavernosa (3) }\end{array}$ & $39-45$ \\
\hline & & & & & & $\begin{array}{l}\text { Fuga dorsal } \\
\text { (1) }\end{array}$ & 43 \\
\hline & & & & & & Sin fugas (1) & 38 \\
\hline & & & & $\begin{array}{l}\text { Curvatura } \\
\text { secundaria } \\
68 / 75 \\
(90,66 \%)\end{array}$ & 58,9 & $\begin{array}{l}\text { Fuga } \\
\text { cavernosa } \\
(26)\end{array}$ & $44-77(62,8)$ \\
\hline & & & & & & $\begin{array}{l}\text { Fuga dorsal } \\
\text { (0) }\end{array}$ & - \\
\hline & & & & & & Sin fugas (42) & $39-68(56,5)$ \\
\hline & & & & $\begin{array}{l}\text { Sin curvatura } \\
2 / 75(1,56 \%)\end{array}$ & 61,5 & $\begin{array}{l}\text { Fuga } \\
\text { cavernosa (2) }\end{array}$ & $61-62$ \\
\hline & & & & & & $\begin{array}{l}\text { Fuga dorsal } \\
(0)\end{array}$ & - \\
\hline & & & & & & Sin fugas $(0)$ & - \\
\hline \multirow[t]{9}{*}{$\begin{array}{l}\text { Moderada } \\
\text { Levine } 223 \\
(17,9 \%)\end{array}$} & 48,4 & $19 / 23(83,6 \%)$ & 53,3 & $\begin{array}{l}\text { Curvatura } \\
\text { primaria } 7 / 23 \\
(30,43 \%)\end{array}$ & 26,4 & $\begin{array}{l}\text { Fuga } \\
\text { cavernosa }(0)\end{array}$ & - \\
\hline & & & & & & $\begin{array}{l}\text { Fuga dorsal } \\
\text { (2) }\end{array}$ & $24-25$ \\
\hline & & & & & & Sin fugas (5) & $16-36(27,2)$ \\
\hline & & & & $\begin{array}{l}\text { Curvatura } \\
\text { secundaria } 3 / \\
23(13,04 \%)\end{array}$ & 62,6 & $\begin{array}{l}\text { Fuga } \\
\text { cavernosa (1) }\end{array}$ & 53 \\
\hline & & & & & & $\begin{array}{l}\text { Fuga dorsal } \\
\text { (0) }\end{array}$ & - \\
\hline & & & & & & Sin fugas (2) & $66-67$ \\
\hline & & & & $\begin{array}{l}\text { Sin curvatura } \\
13 / 23 \\
(56,53 \%)\end{array}$ & 57 & $\begin{array}{l}\text { Fuga } \\
\text { cavernosa (6) }\end{array}$ & $32-68(53,3)$ \\
\hline & & & & & & $\begin{array}{l}\text { Fuga dorsal } \\
\text { (0) }\end{array}$ & - \\
\hline & & & & & & Sin fugas (7) & $47-69(60,1)$ \\
\hline \multirow[t]{9}{*}{$\begin{array}{l}\text { Leve } \\
\text { Levine } 1 \\
30(23,4 \%)\end{array}$} & 44,1 & $20 / 30(66,6 \%)$ & 44,1 & $\begin{array}{l}\text { Curvatura } \\
\text { primaria 3/30 } \\
(10 \%)\end{array}$ & 25 & $\begin{array}{l}\text { Fuga } \\
\text { cavernosa }(0)\end{array}$ & - \\
\hline & & & & & & $\begin{array}{l}\text { Fuga dorsal } \\
\text { (2) }\end{array}$ & $24-26(25)$ \\
\hline & & & & & & Sin fugas (1) & 25 \\
\hline & & & & $\begin{array}{l}\text { Curvatura } \\
\text { secundaria } 0\end{array}$ & - & $\begin{array}{l}\text { Fuga } \\
\text { cavernosa }(0)\end{array}$ & - \\
\hline & & & & & & $\begin{array}{l}\text { Fuga dorsal } \\
\text { (0) }\end{array}$ & - \\
\hline & & & & & & Sin fugas $(0)$ & - \\
\hline & & & & $\begin{array}{l}\text { Sin curvatura } \\
27 / 30(90 \%)\end{array}$ & 46,2 & $\begin{array}{l}\text { Fuga } \\
\text { cavernosa (2) } \\
7,40 \%\end{array}$ & 53 \\
\hline & & & & & & $\begin{array}{l}\text { Fuga dorsal } \\
\text { (10) } 37,03 \%\end{array}$ & $24-48(38,6)$ \\
\hline & & & & & & $\begin{array}{l}\text { Sin fugas (15) } \\
55,5 \%\end{array}$ & $41-60(49,4)$ \\
\hline $\mathrm{n}=128$ & 52,9 & $\mathrm{n}=111$ & & $\mathrm{n}=86$ & & $\mathrm{n}=57$ & \\
\hline
\end{tabular}


162 Cavernosopatía traumática crónica. Un nuevo síndrome de fibrosis peneana Uribe A. y col

DE según grado de fibrosis

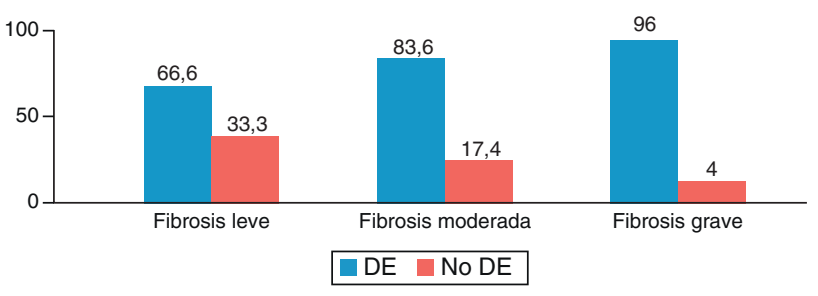

Fig. 5 Disfunción eréctil según el grado de fibrosis.

Tabla 2 Curvaturas primarias vs. curvaturas secundarias

\begin{tabular}{|l|l|l|}
\hline Curvaturas $(\mathbf{n}=\mathbf{8 6} / \mathbf{1 2 8})$ & $\mathbf{N}^{\circ}$ de pacientes & $\%$ \\
\hline Curvatura primaria & 15 & 11,71 \\
\hline Curvatura secundaria & 71 & 55,46 \\
\hline Total de curvaturas & 86 & 67,18 \\
\hline
\end{tabular}

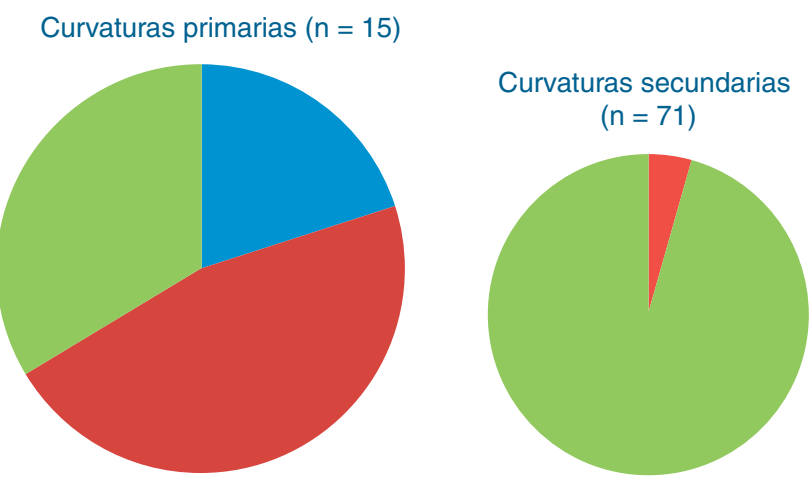

F. leve F. moderada F. grave

Fig. 6 Fibrosis según la curvatura.

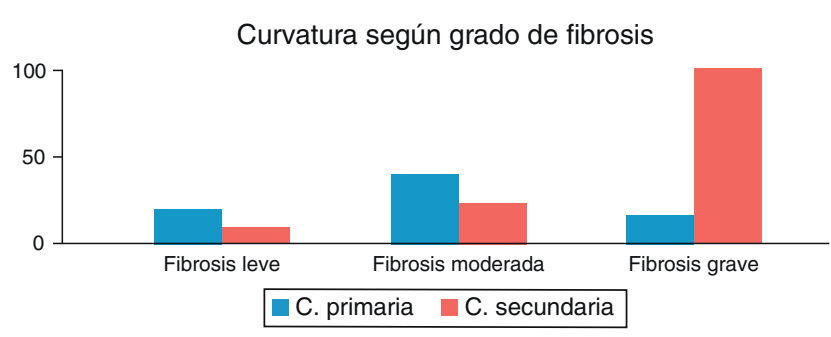

Fig. 7 Curvaturas en los diferentes grados de fibrosis.

mayor (59,10 años) que el grupo con «menor posibilidad de CTC» con 46 pacientes $(35,97 \%)$ y un promedio de edad de 35,6 años (-Tabla 6).

\section{Discusión y Análisis}

Este estudio aporta una idea novedosa: la fibrosis peneana extratunical es mucho más frecuente que la enfermedad de Peyronie, lo que contradice a los autores que han señalado que dicha fibrosis en la Peyronie (usualmente buscada como
Tabla 3 Fugas vs. no fugas y fuga dorsal vs. fuga cavernosa

\begin{tabular}{|l|l|l|}
\hline Fugas venosas $(\mathbf{n}=\mathbf{5 7 / 1 2 8})$ & $\mathbf{N}^{\circ}$ de pacientes & $\%$ \\
\hline Fugas cavernosas & 44 & 34,37 \\
\hline Fuga dorsal & 15 & 11,71 \\
\hline Total fugas & 57 & 46,08 \\
\hline
\end{tabular}

Fugas dorsales $(n=15)$

Fugas cavernosas $(n=42)$
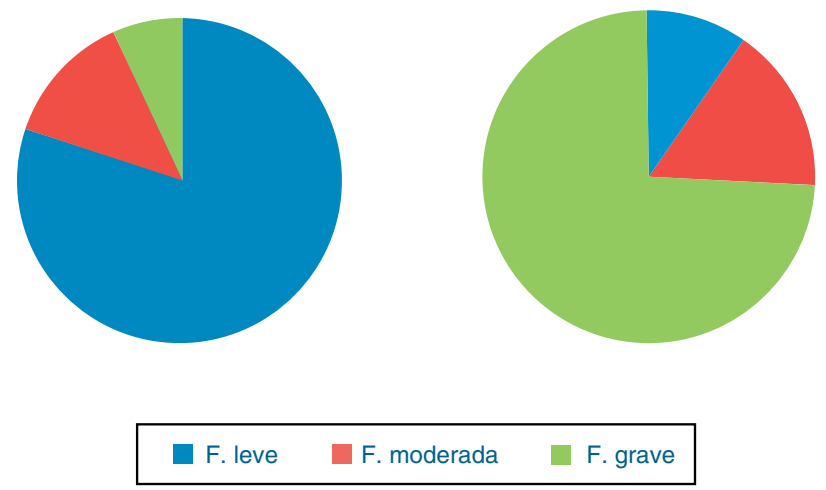

Fig. 8 Grado de fibrosis según la fuga.

Fugas según grado de fibrosis

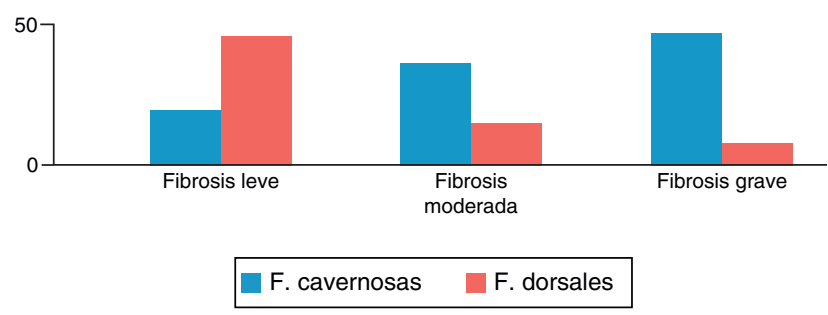

Fig. 9 Fugas en los diferentes grados de fibrosis.

una placa palpable o ultrasonográfica que compromete necesariamente la túnica albugínea), se encuentra apenas en entre el 20 y el 55\% de los pacientes con fibrosis peneana, teniendo en cuenta que las fibrosis leves o moderadas son subdiagnosticadas. ${ }^{11,23-25}$

La Peyronie es una condición fibrosa que clínicamente tiene curvaturas secundarias, placas palpables y ultrasonográficas, indentaciones y otras deformidades del pene, calcificaciones, osificación, con relativamente poca disfunción eréctil asociada.

Las teorías que intentan explicar la enfermedad fibrosa peneana del tipo Peyronie muestran un proceso inflamatorio crónico de etiología multifactorial. Una línea defiende el origen genético, con factores que inducen, facilitan o desencadenan bajo ciertas condiciones la aparición de fibrosis. Esa predisposición genética se ha visto relacionada con duplicaciones o trisomías de los cromosomas 7 y 8 , deleciones del cromosoma $\mathrm{Y}$ y otras muchas más. Es conocida también la asociación entre la Peyronie genética y las enfermedades de Dupuytren o Ledderhose, hasta en un $20 \%$ de los casos, pero lo cierto es que, comparado con el 
Tabla 4 Resumen de los diferentes hallazgos de la CTC

\begin{tabular}{|c|c|c|c|c|}
\hline & $\begin{array}{l}\text { Fibrosis leve } \\
\text { Levine } 1(n=30)\end{array}$ & $\begin{array}{l}\text { Fibrosis moderada } \\
\text { Levine } 2(n=23)\end{array}$ & $\begin{array}{l}\text { Fibrosis grave } \\
\text { Levine } 3(n=75)\end{array}$ & $\mathrm{p}$ \\
\hline Disfunción eréctil ( $\mathrm{n}=111) \mathrm{n}(\%)$ & $20(18,0)$ & $19(17,1)$ & $72(64,9)$ & 0,0001 \\
\hline RP (IC 95\%) & * & $1,24(0,90-1,70)$ & $1,44(1,11-1,86)$ & \\
\hline Curvatura primaria $(\mathrm{n}=15) \mathrm{n}(\%)$ & $3(20)$ & $7(46,6)$ & $5(33,3)$ & 0,0006 \\
\hline RP (IC 95\%) & * & $3,5(1,0-12,0)$ & $7,1(2,2-23,0)$ & \\
\hline Curvatura secundaria $(n=71) n(\%)$ & 0 & $3(4,2)$ & $68(95,7)$ & $<0,0001$ \\
\hline RP (IC 95\%) & NA & $0,2(0,1-0,5)$ & 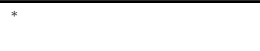 & \\
\hline Fuga cavernosa $(n=42) n(\%)$ & $4(9)$ & $9(20,4)$ & $31(70,4)$ & 0,0060 \\
\hline RP (IC 95\%) & ${ }^{*}$ & $2,3(0,8-6,9)$ & $3,1(1,2-8,0)$ & \\
\hline
\end{tabular}

Abreviaciones: CTC, cavernosopatía traumática crónica; IC, intervalo de confianza.

Tabla 5 Pacientes según cumplimiento de criterios de CTC, desde 4 (todos) a 1 criterio; énfasis en pacientes con curvatura secundaria y fugas cavernosas y su relación con la edad

\begin{tabular}{|l|l|l|l|l|}
\hline Criterios de CTC $(\mathbf{n}=\mathbf{1 2 8})$ & \multicolumn{2}{|l|}{$\mathbf{0 , 2}$-3] Discriminación de criterios } & \% parcial & Edad promedio en años \\
\hline Cuatro $(n=35)$ & $\begin{array}{l}\text { Con curva secundaria }+ \\
\text { fuga cavernosa }\end{array}$ & 27 & 21,09 & 62,51 \\
\hline & $\begin{array}{l}\text { Con curva primaria } \\
\text { o fuga dorsal }\end{array}$ & 8 & 6,24 & 33,5 \\
\hline Tres $(n=67)$ & 43 (sin fuga) & Curva secundaria: 41 & 32,03 & 57,95 \\
\hline & & Curva primaria: 2 & 1,56 & 37 \\
\hline & 24 (sin curva) & Fuga dorsal: 10 & 7,81 & 38,6 \\
\hline & & Fuga cavernosa: 14 & 10,93 & 56,85 \\
\hline Dos $(n=17)$ & $\begin{array}{l}\text { (sin disfunción } \\
\text { eréctil y fuga) }\end{array}$ & Curva secundaria: 3 & 2,34 & 25 \\
\hline & & Curva primaria: 5 & 2,77 & 44,2 \\
\hline & 9 (sin curva y fuga) & & 8,17 & 56,22 \\
\hline Uno $(n=9)$ & $\begin{array}{l}9 \text { (sin disfunción eréctil, } \\
\text { curva y fuga) }\end{array}$ & & 7,03 & 47,22 \\
\hline
\end{tabular}

Abreviaciones: CTC, cavernosopatía traumática crónica.

Criterios de CTC

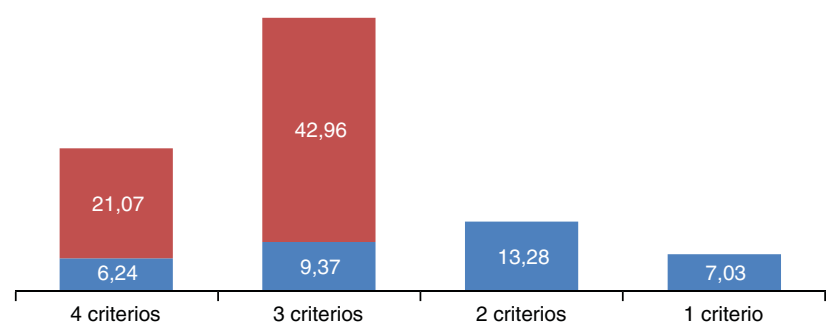

Menor $\square$ Mayor
Tabla 6 Pacientes con alto riesgo de CTC (al menos 3 criterios, y que estos incluyan curvatura secundaria o fuga cavernosa)

\begin{tabular}{|l|l|l|l|}
\hline CTC $\mathbf{n}=128$ & $\%$ & Edad en años & DE \\
\hline $\begin{array}{l}\text { Mayor posibilidad de } \\
\text { CTC }(\mathbf{n}=79)\end{array}$ & 64,03 & 59,10 & 8,7 \\
\hline $\begin{array}{l}\text { Menor posibilidad de } \\
\text { CTC }(\mathrm{n}=49)\end{array}$ & 35,97 & 35,66 & 11,7 \\
\hline
\end{tabular}

Abreviaciones: CTC, cavernosopatía traumática crónica; DE, desviación estándar.

evoluciona a fibrosis de diversos grados y cambios en la matriz extracelular, en individuos que pueden o no estar genéticamente predispuestos. ${ }^{12,24,25,28-42}$

En el presente estudio se han propuesto 2 escenarios potenciales que generan fibrosis: el secundario a traumas mayores del pene (excluidos en este estudio) y el de la fibrosis secundaria, muy posiblemente relacionada con MSR, que fueron incluidos. 
Como fuente de esos microtraumas existen 2 opciones reconocidas: los secundarios a erección parcial (menor de $7 / 10$ ), que implican debilitar un instrumento rectilíneo que debe vencer las fuerzas axiales que se le oponen en vagina 0 ano, y los secundarios a curvaturas mayores de $30^{\circ}$; ambos escenarios angulan (o hiperangulan) el pene durante la penetración y generan un roce traumático (MSR) contra las paredes de la vagina, lo que potencialmente genera un daño o noxa que se traduce en fibrosis ulterior.

La segunda causa de MSR es el generado por curvaturas peneanas con 2 escenarios: curvaturas primarias usualmente congénitas que ocurren por un desequilibrio en el crecimiento entre los cuerpos cavernosos y que tienen posibilidad de desarrollar CTC hacia el futuro por ser una causa de microtrauma repetido (consideradas en este estudio) y curvaturas secundarias como consecuencia de las zonas de fibrosis aledañas (consideradas también en este estudio).

Una gran disyuntiva nos creó el dato de cómo considerar a los pacientes con curvaturas primarias: sanos actuales, que potencialmente harán otras curvaturas secundarias hacia el futuro debido al trauma crónico (CTC) o enfermos, en camino de una CTC por su factor de riesgo, al estar curvados. Al hacerlo de esta manera nos encontramos con el dato aplastante de un $90 \%$ de curvaturas secundarias en el grupo de fibrosis grave (L3) comparado con el $8,22 \%$ en el grupo de curvaturas primarias o sin curvaturas. Es casi inherente a la fibrosis grave (aun sin ser Peyronies clásicos) la curvatura secundaria.

Un dato interesante en el pequeño grupo de curvaturas primarias $(n=15)$ es la preponderancia de la fibrosis moderada (L2), casi en el 50\%; aunque el número de pacientes es muy pequeño, podría inferirse que la curvatura primaria sí es una puerta de entrada para mayores grados de fibrosis por MSR, es decir CTC; sin embargo, este ítem ameritaría un estudio prospectivo, puesto que algunas curvaturas que comenzaron como primarias se modificarían en el tiempo por el CTC, empeorando y creando una nueva categoría que no está considerada y que podría llamarse "mixta» (curvatura primaria que empeoró o se modificó y se convirtió en secundaria). ${ }^{43}$

Las teorías que explican la aparición de fugas venosas consideradas en el estudio son 2: fugas venosas secundarias, usualmente intracavernosas, que aparecen al lado de las zonas de fibrosis principal, que hemos llamado "fuga índice», y fugas venosas primarias usualmente congénitas, originadas desde la vena dorsal profunda hacia los plexos periprostáticos y que causan fallas eréctiles, que pueden cursar incluso con disfunción eréctil primaria y que fueron consideradas en este estudio por su capacidad de convertirse en un factor inductor de MSR, acumulador de fibrosis, desde muy temprano. En este estudio, al comparar a los individuos, tenían mayor riesgo de fuga cavernosa al empeorar la fibrosis: 70,$4 ; 20,4$ y $9 \%$ para L3, L2 y L1, respectivamente.

Se debe aceptar que los datos del estudio implican un tipo de sesgo en la escogencia, puesto que los pacientes con fibrosis grave tenían mayor posibilidad de ser enviados a una ecografía dopler de pene con vasoactivo, criterio de inclusión fundamental; al comparar los datos del número de pacientes por grado en este estudio frente al artículo original de Levine cuando publicó su clasificación, se encuentra que: grado 1 (23,4 vs. $40,8 \%)$; grado 2 (17,9 vs. $27,5 \%$ ) y grado 3 (56,5 vs. $31,6 \%$ ) en CTC vs. Levine, respectivamente. Es decir, nuestro grupo tenía más graves y menos leves que Levine. Ni el estudio de Levine ni este, que es retrospectivo, separaron en 2 grupos las fibrosis extralbugíneas (CTC) de las intralbugíneas (Peyronie). ${ }^{11,18}$

Aunque ya se mencionó que son enfermedades distintas, haciendo una analogía con la Peyronie, existen al menos

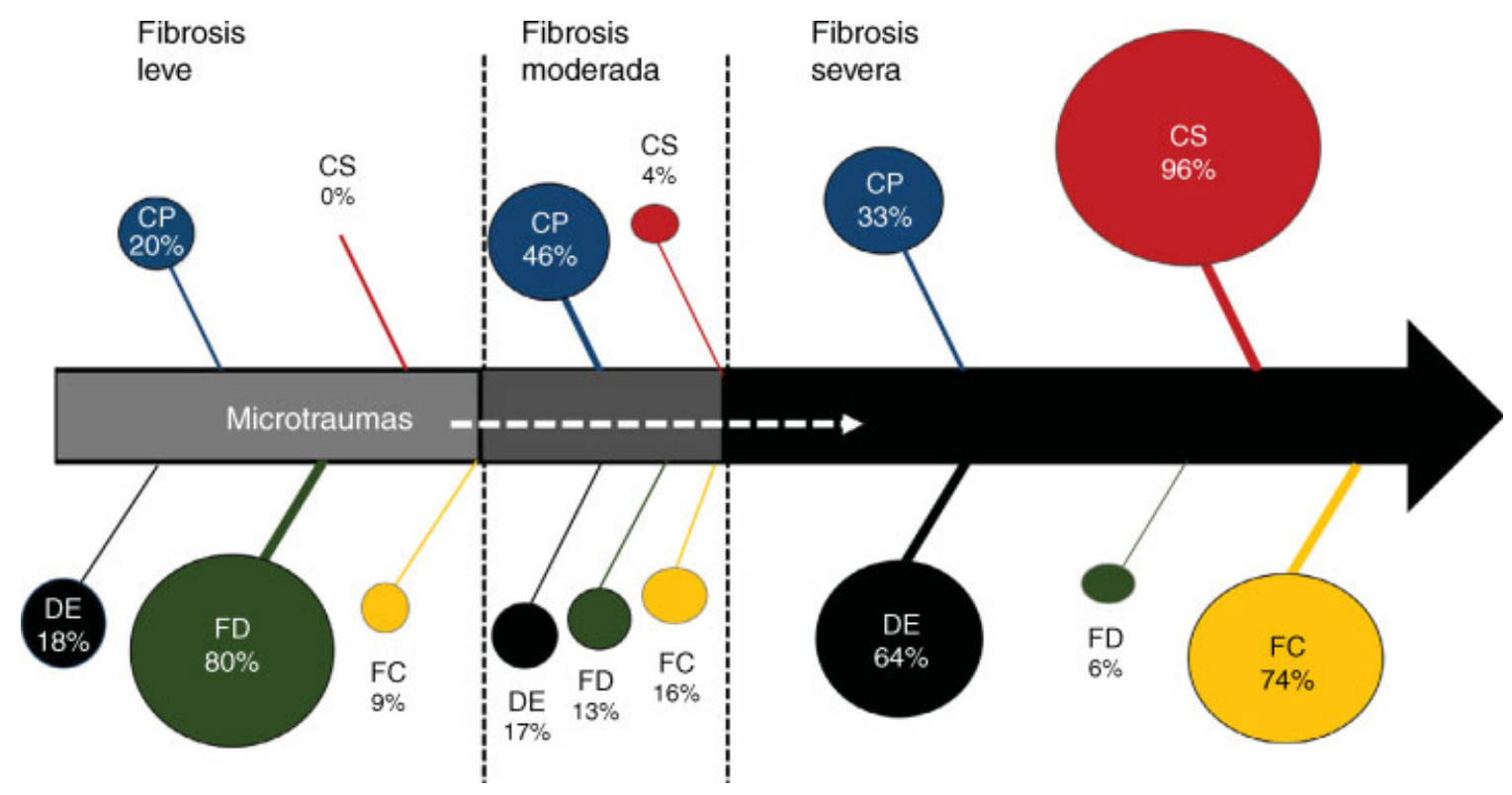

Fig. 11 Resumen de los criterios de CTC según el peso de cada ítem. Abreviaciones: CP: curvatura primaria; CS: curvatura secundaria; DE: disfunción eréctil; FC: fuga cavernosa; FD: fuga dorsal. 
4 desenlaces posibles para esta enfermedad: desaparición espontánea (rara); estabilidad de la placa sin curvatura (poco frecuente); estabilidad de la placa con curvatura (usualmente creciente) e inestabilidad con máxima fibrosis (calcificación, osificación) y curvaturas sucesivas, habitualmente con deformidad (retracción, reloj de arena, etc.). El desenlace de los pacientes de CTC es empeoramiento de la fibrosis, fugas venosas relacionadas, curvaturas secundarias y disfunción eréctil progresiva (- Fig. 11). ${ }^{11,37,44-52}$

\section{Conclusiones}

Proponemos la existencia de un síndrome de MSR en el pene que puede denominarse CTC con 4 componentes: fibrosis cavernosa extratunical, disfunción eréctil, curvatura peneana y fugas venosas.

La fibrosis cavernosa es un continuum. Antes de alcanzar el grado de fibrosis grave, que se ha conocido genéricamente como «enfermedad de Peyronie» cuando está comprometida la túnica albugínea, el paciente trascurre por fases de fibrosis leve y moderada que pasan a menudo desapercibidas para los clínicos, puesto que su diagnóstico es por imágenes.

La disfunción eréctil tiene relación directa con fugas cavernosas y con la fibrosis grave.

Las curvaturas secundarias y las fugas cavernosas están relacionadas directamente con el grado de fibrosis.

Las curvaturas primarias son una puerta de entrada a la fibrosis, incluso grave.

La edad agrava todos los componentes de la CTC.

Existe un nuevo papel para la ecografía dopler del pene con vasoactivo como una manera efectiva de clasificar la fibrosis peneana con posibles implicaciones en las guías de manejo.

\section{Recomendaciones}

Es necesario hacer un estudio prospectivo sobre el tema de la CTC.

En el futuro se deberían tipificar anatomopatológicamente los hallazgos ecográficos de los diferentes tipos de fibrosis.

El presente estudio indica que existe un nuevo síndrome de fibrosis peneana denominado CTC. En el se incluyó a pacientes con enfermedad de Peyronie, especialmente en el grupo de fibrosis grave o L3. Todo estudio futuro debería diferenciar claramente los 2 grupos, puesto que son distintos.

\section{Responsabilidades éticas}

\section{Protección De Personas Y Animales}

Los autores declaran que para esta investigación no se han realizado experimentos en seres humanos ni en animales.

\section{Confidencialidad de los datos}

Los autores declaran que en este artículo no aparecen datos de pacientes.

\section{Derecho a la privacidad y consentimiento informado} Los autores declaran que en este artículo no aparecen datos de pacientes.
Conflicto de intereses

Ninguno.

\section{Bibliografía}

1 Tefekli FA, Kandirali FE, Erol FH, Alp FT, Koksal FT, Kadioglu FA. Peyronie's disease in men under age 40: Characteristics and outcome. Int J Impot Res 2001;13:18

2 De le Peyronie F. Sur quel ques obstacles quis'opposent à l'éjaculation naturelle de la semence. Mem Acad Roy Chir 1743; 1:425-434

3 Tal FR, Hall FM, Alex FB, Choi FJ, Mulhall FJ. Peyronie's disease in teenagers. J Sex Med 2012;9:302

4 Levine FL, Estrada FC, Storm FD, Matkov FT. Peyronie disease in younger men: Characteristics and treatment results. J Androl 2003;24:27-32

5 Levine FL, Estrada FC, Storm FD, Matkov FT. Peyronie disease in younger men: Characteristics and treatment results. J Androl 2003;24:27-32 [PubMed PMID: 12514077]

6 Brenot FP. Male impotence. A historical perspective. París: L'Esprit du Temps; 1994:46-53

7 A. Martín Morales, V. Chantada Abal, N. Cruz Navarro, L. Fiter Gómez, L. Rodríguez Vela, I. Moncada Iribarren. Enfermedad de La Peyronie y otras alteraciones morfométricas del pene. Asociación Española de Urología, Tema Monográfico del LXVI Congreso Nacional de Urología, Zaragoza (España), mayo de 2001. Disponible en: http://www.aeu.es/UserFiles/File/Peyronie.pdf

8 Hauck FE, Weidner FW. François de la Peyronie and the disease named after him. Lancet 2001;357:2049-2051 [PubMed PMID: 11438159]

9 Dunsmuir FW, Kirby FR. Francois de la Peyronie (1678-1747): The man and the disease he described. Br J Urol 1996;78:613-622

10 Ferretti FL, Fandel FT, Qiu FX et al. Tunica albuginea allograft: A new model of La Peyronie's disease with penile curvature and subtunical ossification. Asian J Androl 2014;16:592-596

11 Laurence FA. Levine, and Stephen Larsen. Cap 31. Diagnosis and management of Peyronie disease. In: Wein FA, Kavoussi FL, Partin FA. Peters FC. Campbell-Walsh Urology, 11th edition. Elsevier: Philadelphia; 2016:722-748

12 Omalu FB, DeKosky FS, Minster FR, Kamboh FM, Hamilton FR, Wecht FC. Chronic traumatic encephalopathy in a National Football League player. Neurosurgery 2005;57:128-134 [discussion 128-34. PubMed PMID: 15987548]

13 Turner FR, Lucke-Wold FB, Logsdon FA et al. The questto model chronic traumatic encephalopathy: A multiple model and injury paradigm experience. Front Neurol 2015;6:222

14 Chung FE, Ralph FD, Kagioglu FA, et al. Evidence-based management guidelines on Peyronie's disease. J Sex Med 2016;13:905-923

15 Berookhim FB. Doppler duplex ultrasonography of the penis. Sex Med 2016;13:726e731

16 Broderick FG. Valoración vascular de la disfunción eréctil. In: Lue FT. Atlas de la disfunción sexual masculina. San Francisco: Current Medical Group; 2008

17 Gilbert BR, Paduch D. Penile dopler ultrasound. Post graduate course. En: American Urological Association (AUA), Annual Meeting; Orlando (FL), 17 de mayo de 2014

18 Levine FL, Rybak FJ, Corder FC, Farrel FM. Peyronie's disease plaque calcification-prevalence, time to identification, and development of a new grading classification. J Sex Med 2013;10:3121-3128

19 Levine FL, Coogan FC. Penile vascular assessment using color duplex sonography in men with Peyronie's disease. J Urol 1996; 15:1270-1273

20 Levine FL, Greenfield FJ. Establishing a standardized evaluation of the man with Peyronie's disease. Int J Impot Res 2003;15(Suppl 5): S103-S112

21 Yuruk FE, Serefoglu FE. Re Peyronie's disease plaque calcificationprevalence, time to identification, and development of a new 
grading classification. J Sex Med 2014;11:1351 [C PubMed PMID: 24877175]

22 Bekos FA, Arvaniti FM, Hatzimouratidis FK, Moysidis FK, Tzortzis FV, Hatzichristou FD. The natural history of Peyronie's disease: An ultrasonography-based study. Eur Urol 2008;53:644-650 [Epub 2007 Jul 17. PubMed PMID: 17673362]

23 Peyronie disease: AUA guidelines 2015 [consultado 18 May 2016]. Disponible en: www.auanet.org/education/guidelines/peyroniedisease.cfm

24 Smith FB. Peyronie's disease. Am J Clin Pathol 1966;45:670-678

25 Choe FS, Veliceasa FD, Bond FC, et al. Sonic hedgehog delivery from self-assembled nanofiber hydrogels reduces the fibrotic response in models of erectile dysfunction. Acta Biomater 2016;32:89-99

26 Brock FG. Editorial comment: Peyronie's disease and autoimmunity -a real-life clinical study and comprehensive review. J Sex Med 2015;12:1071

27 Ventimiglia FE, Capogrosso FP, Colicchia FM, et al. Peyronie's disease and autoimmunity-a real life clinical study and comprehensive review. J Sex Med 2015;12:1062

28 Levine FL, Burnett FA. Standard operating procedures for Peyronie's disease. J Sex Med 2013;10:230-244 [doi: 10.1111/j. 1743-6109.2012.03003.x. Epub 2012 Dec 4. Review. PubMed PMID: 23211057]

29 Taylor FF, Levine FL. Peyronie's disease. Urol Clin North Am 2007; 34:517-534 [vi. Review. PubMed PMID: 17983892]

30 Herati FA, Pastuszak FA. The genetic basis of Peyronie's disease: A review. Sex Med Rev 2016;4:85-94

31 Nugteren FH, Nijman FJ, de Jong FI, van Driel FM. The association between Peyronie's and Dupuytren's disease. Int J Impot Res 2011;23:142-145

32 Nyberg FL, Bias FW, Hochberg FM, Walsh FP. Identification of an inherited form of Peyronie's disease with autosomal dominant inheritance and association with Dupuytren's contracture and histocompatibility B7 cross-reacting antigens.J Urol 1982;128:48-51

33 Zorba FO, Sirma FS, Ozgon FG, Salabas FE, Ozbek FU, Kadioglu FA. Comparison of apoptotic gene expression profiles between Peyronie's disease plaque and tunica albuginea. Adv Clin Exp Med 2012;21:607

34 Hinman FF. Etiologic factors in Peyronie's disease. Urol Int 1980; 35:407-113

35 Silva FJ, Rodríguez FS, Sáenz FM. Actualización sobre la enfermedad de Peyronie univ. Méd Bogotá (Colombia) 2010;51:320-327

36 Vernet FD, Nolazco FG, Cantini FL, et al. Evidence that osteogenic progenitor cell in the human tunica albuginea may originate from stem cell: Implications for Peyronie disease. Biol Reprod 2005; 73:1199-1210

37 Kadioglu FA, Sanli FO, Akman FT, et al. Factors affecting the degree of penile deformity in Peyronie disease: An analysis of 1001 patients. J Androl 2011;32:502-508

38 Kalokairinou FK, Konstantinidis FC, Domazou FM, Kalogeropoulos FT, Kosmidis FP, Gekas FA. US imaging in Peyronie's disease. J Clin Imaging Sci 2012;2:63
39 Graziottin FT. The pathophysiology of Peyronie's disease: Beyond the Smith's space. Int Braz J Urol 2015;41:1040-1042 [doi: 10.1590/S1677-5538.IBJU.2015.06.02. PubMed PMID: 26717114]

40 Iacono FF, Giannella FR, Somma FP, Manno FG, Fusco FF, Mirone FV. Histological alterations in cavernous tissue after radical prostatectomy. J Urol 2005;173:1673-1676

41 Teflekli FA, Kandiarali FE, Erol FB, Tunc FM, Kadioglu FA. Peyronie's disease: A silent consequence of diabetes mellitus. Asian J Androl 2006;8:75-79

42 Pryor FJ, Castle FW. Peyronie's disease associated with chronic degenerative arterial disease and not with beta-adrenoceptor blocking agents. Lancet 1982;1:917

43 Gelbard FM. Dystrophic penile calcification in Peyronie's disease. J Urol 1988;139:738-740

44 Serefoglu FE, Trost FL, Sikka FS, Hellstrom FW. The direction and severity of penile curvature does not have an impact on concomitant vasculogenic erectile dysfunction in patients with Peyronie's disease. Int J Impot Res 2015;27:6-8

45 Kelami FA. Classification of congenital and acquired penile deviation. Urol Int 1983;38:229-233

46 Gonzalez-Cadavid FN, Rajfer FJ. Mechanisms of disease: New insights into the cellular and molecular pathology of Peyronie's disease. Nat Clin Pract Urol 2005;2:291-297 [Review. PubMed PMID: 16474811]

47 Ferrini FM, Kovanecz FI, Sanchez FS, Umeh FC, Rajfer FJ, GonzalezCadavid FN. Fibrosis and loss of smooth muscle in the corpora cavernosa precede corporal veno-occlusive dysfunction (CVOD) induced by experimental cavernosal nerve damage in the rat. J Sex Med 2009;6:415-428

48 Luo FH, Goldstein FI, Udelson FD. A three-dimensional theoretical model of the relationship between cavernosal expandability and percent cavernosal smooth muscle. J Sex Med 2007;4:644-645 [PubMed: 17498102]

49 Nikoobakht FM, Saraji FA, Meysamie FA. Preoperative corporal biopsy as a predictor of postoperative results in venoocclusive erectile dysfunction. Urol J 2005;2:160-164

50 Iacono FF, Barra FS, De Rosa FG, Boscaino FA, Lotti FT, Microstructural disorders of tunica albuginea in patients affected by Peyronies's disease with or with out erection dysfunction. J Urol 1993;150:1806-1809

51 Shafik FA, Shafik FI, El Sibai FO, Shafik FA. On the pathogenesis of penile venous leakage: Role of the tunica albuginea. BMC Urol 2007;7:14 [PubMed PMID: 17803807; PubMed Central PMCID: PMC1995196]

52 Hsu FG, Hsieh FC, Wen FH, et al. Anatomy of the human penis: The relationship of the architecture between skeletal and smooth muscles. J Androl 2004;25:426-431

53 El-Sakka FA, Yassin FA. Amelioration of penile fibrosis: Myth or reality. J Androl 2010;31:324-335

54 Traish FA, Toselli FP, Jeong FS, Kim FN. Adipocyte accumulation in penile corpus cavernosum of the orchiectomized rabbit: A potential mechanism for veno-occlusive dysfunction in androgen deficiency. J Androl 2005;26:242-48 